\section{Anatomy of the posterior approach to the lumbar plexus block}

Juliana Farny MD, ${ }^{*}$ Pierre Drolet MD FRCPC, $\uparrow$ Michel Girard MD MHPE FRCPC $\dagger$
The purpose of this study was to describe the relation of the lumbar plexus with the psoas major and with the superficial and deep landmarks close to it. Four cadavers were dissected and 22 computed tomography files of the lumbasacral region studied. Cadaver dissections demonstrated that the lumbar plexus, at the level of $L_{5}$, is within the substance of the psoas major muscle rather than between this muscle and the quadratus lumborum. The femoral nerve lies between the lateral femoral cutaneous and obturator nerves. However, while the lateral femoral cutaneous nerve is in the same fascial plane as the femoral nerve, the obturator nerve can be found in the same plane as the two other nerves or in its own muscular fold. Radiological data provided the following measurements: the femoral nerve is at a depth of $9.01 \pm 2.43 \mathrm{~cm}$; the psoas major medial border is at $2.73 \pm 0.64 \mathrm{~cm}$ from the median sagital plane; and its lateral border is at $6.41 \pm 1.61 \mathrm{~cm}$ from the same plane. It is concluded that the lumbar plexus is within the psoas major, that the obturator nerve localization within the psoas major varies and that computed tomography data define precisely the relationship of the lumbar plexus with superficial and deep landmarks.

Le but de ce travail était de décrire la relation anatomique entre le plexus lombaire et le muscle psoas, de même qu'avec des repères anatomiques superficiels et profonds. Quatre cadavres ont été disséqués et 22 tomographies de la région lombosacrée étudiées. Les dissections montrent que le plexus lombaire, au niveau de $L_{g}$, se trouve dans le muscle psoas plutôt

\section{Key words}

ANATOMY: lumbar plexus;

ANAESTHETIC TECHNIQUES: regional, lumbar plexus block, sciatic nerve block.

From the Département d'anesthésie-réanimation, †Hôpital Maisonneuve-Rosemont et Université de Montréal, Montréal, Québec and *CHRU de Strasbourg, France.

Address correspondence to: Dr Pierre Drolet, Département d'anesthésie-réanimation, Hôpital Maisonneuve-Rosemont, 5415 boul. L’Assomption, Montréal, Québec, H1T 2M4. Accepted for publication 3rd March, 1994. qu'entre celui-ci et le carré des lombes. Le nerf fémoral est situé entre les nerfs fémoro-cutané latéral et obturateur. Cependant, alors que le nerf fémoro-cutané latéral est dans le même plan que le nerf fémoral, le nerf obturateur peut les y accompagner ou se retrouver dans un repli du muscle psoas qui le sépare des deux autres nerfs. Les études radiologiques ont fourni les données suivantes: le nerf fémoral est à une profondeur de $9,01 \pm 2,43 \mathrm{~cm}$; le bord interne du psoas est à $2,73 \pm 0,64 \mathrm{~cm}$ du plan sagital médian; alors que le bord externe de ce muscle est à $6,41 \pm 1,61 \mathrm{~cm}$ de ce même plan. Nous concluons que le plexus lombaire est dans le muscle psoas, que la position du nerf obturateur varie par rapport au nerf fémoral et que la tomographie aide à situer le plexus par rapport à d'autres repères.

A knowledge of the anatomy is essential to an anaesthetist who wishes to perform a regional anaesthesia techique and the posterior approach to the lumbar plexus is no exception. The literature on lumbar plexus anatomy contains contradictory information. Winnie ${ }^{1}$ and Wedel $^{2}$ locate the lumbar plexus between the quadratus lumborum and psoas major muscles. However, a number of anatomy textbooks, ${ }^{3-6}$ Chayen ${ }^{7}$ and more recently a radiological study by Dietemann and Sick ${ }^{8}$ place the lumbar plexus within the substance of the psoas major.

The goal of the study was to describe precisely the anatomical course of the lumbar plexus and its relationship with adjacent structures and with surface and deep anatomical landmarks.

\section{Methods}

The protocol was reviewed and approved by the research and ethics committee of our institution. This work consists of two parts. In the first, four adult fresh cadavers (two women and two men) were dissected to describe the topography of the lumbar plexus and its branches. After the abdominal cavity was emptied of viscera, a meticulous dissection of the two psoas major muscles between $L_{2}-L_{3}$ and $S_{1}-S_{2}$ was carried. The lumbar plexus and its branches relationships with other landmarks were noted. Once the dissection was finished, the muscles were 


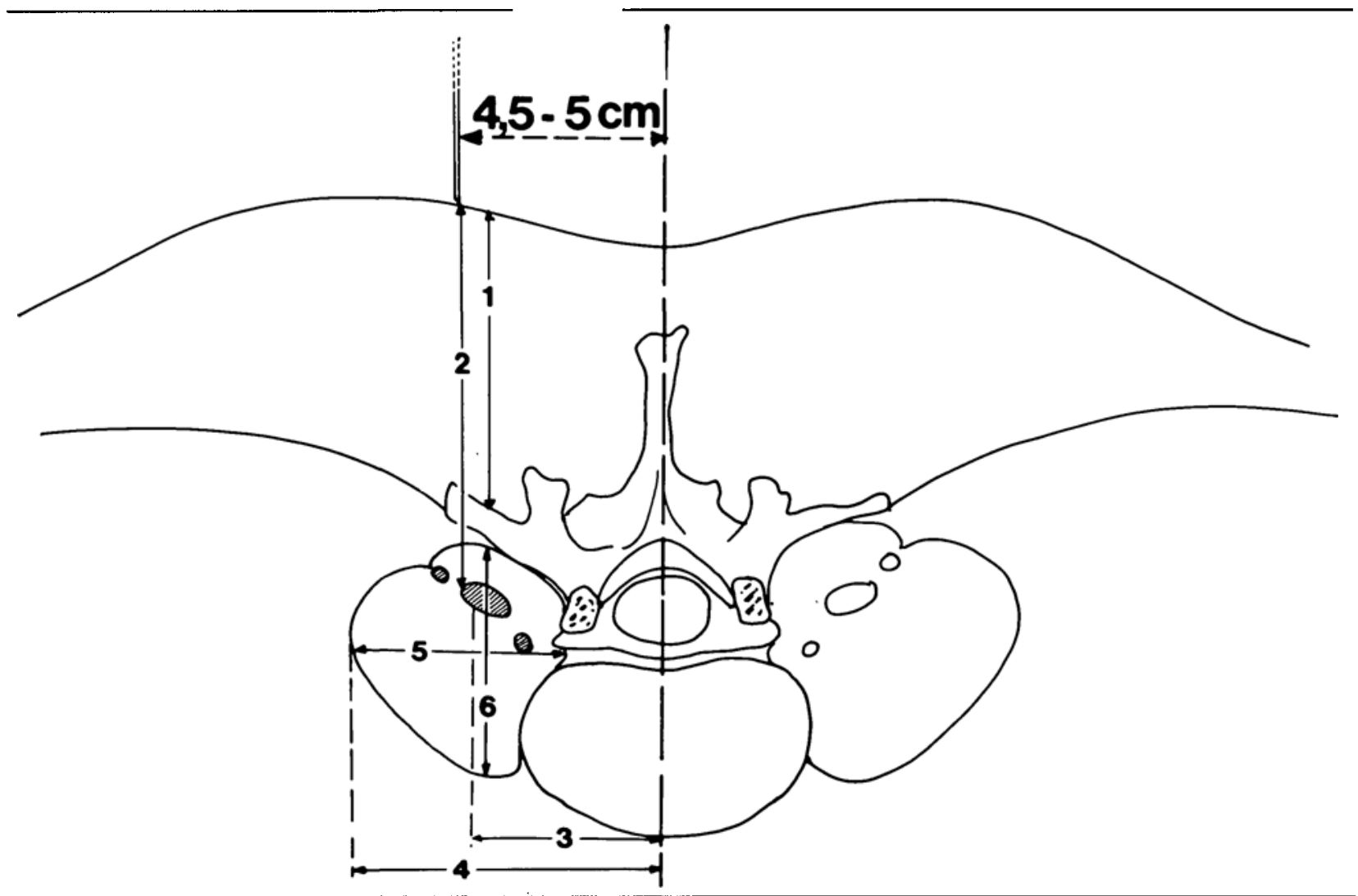

FIGURE 1 Dissection data collection landmarks. (1) Distance skin/transverse process $\mathrm{L}_{5}$, (2) distance skin/lumbar plexus nervous trunks, (3) distance nervous trunks/median sagital plane, (4) distance psoas major lateral border/median sagital plane, (5) psoas major frontal diameter, (6) psoas major median sagital diameter.

placed in their original position and a transverse section was made at the level of the $\mathrm{L}_{4-5}$ intervertebral disc. Measurements (Figure 1) were made from the transverse section.

In the second part, axial transverse sections of computed tomography of the lumbosacral region $\left(\mathrm{L}_{1}\right.$ to $\left.\mathrm{S}_{2-3}\right)$ were used to compute the following measurements and distances: subcutaneous fat thickness, intervertebral disc diameter, distance of the skin to the transverse process, distance of the medial aspect of the psoas to the median sagital plane, distance of the lateral aspect of the psoas to the median sagital plane, distance of the posterior aspect of the psoas to the skin, distance of the anterior aspect of the psoas to the skin and the estimated distance of the femoral nerve to the skin. The radiological documents were from 22 adults (13 women and nine men) studied for various pathological conditions. All patients had a normal lumbosacral region.

\section{Results}

\section{Cadaver dissection}

The lumbar plexus (Figure 2) originated from the first four lumbar ventral rami that joined within the substance of the psoas major. The plexus displayed a triangular shape, narrow in its superior portion and wider in its lower portion. It passed anteriorly to the lumbar transverse processes within the substance of the psoas major. It lay between: (a) the fleshy slips of the main part of the psoas major that arose from the antero-lateral part of the vertebral bodies and the intervertebral disc and (b) the accessory part of the psoas, posterior to the main part of the muscle, that originated from the anterior part of the transverse processes. The two parts of the muscle fused to form the psoas major. However, near the vertebral bodies, these two parts were separated by a thin fascia within which were the roots of the lumbar plexus and the ascending lumbar vein. The first lumbar ventral ramus divided to give the iliohypogastric and ilioinguinal nerves that left the psoas muscle to travel anteriorly to the quadratus lumborum. It is noteworthy that these nerves were the only elements of the lumbar plexus in contact with this muscle. The genitofemoral and the lateral femoral cutaneous nerves originated from the second lumbar ventral ramus. The first descended on the ventral aspect of the psoas major muscle while the latter crossed the lateral border of the psoas major at the level of the 


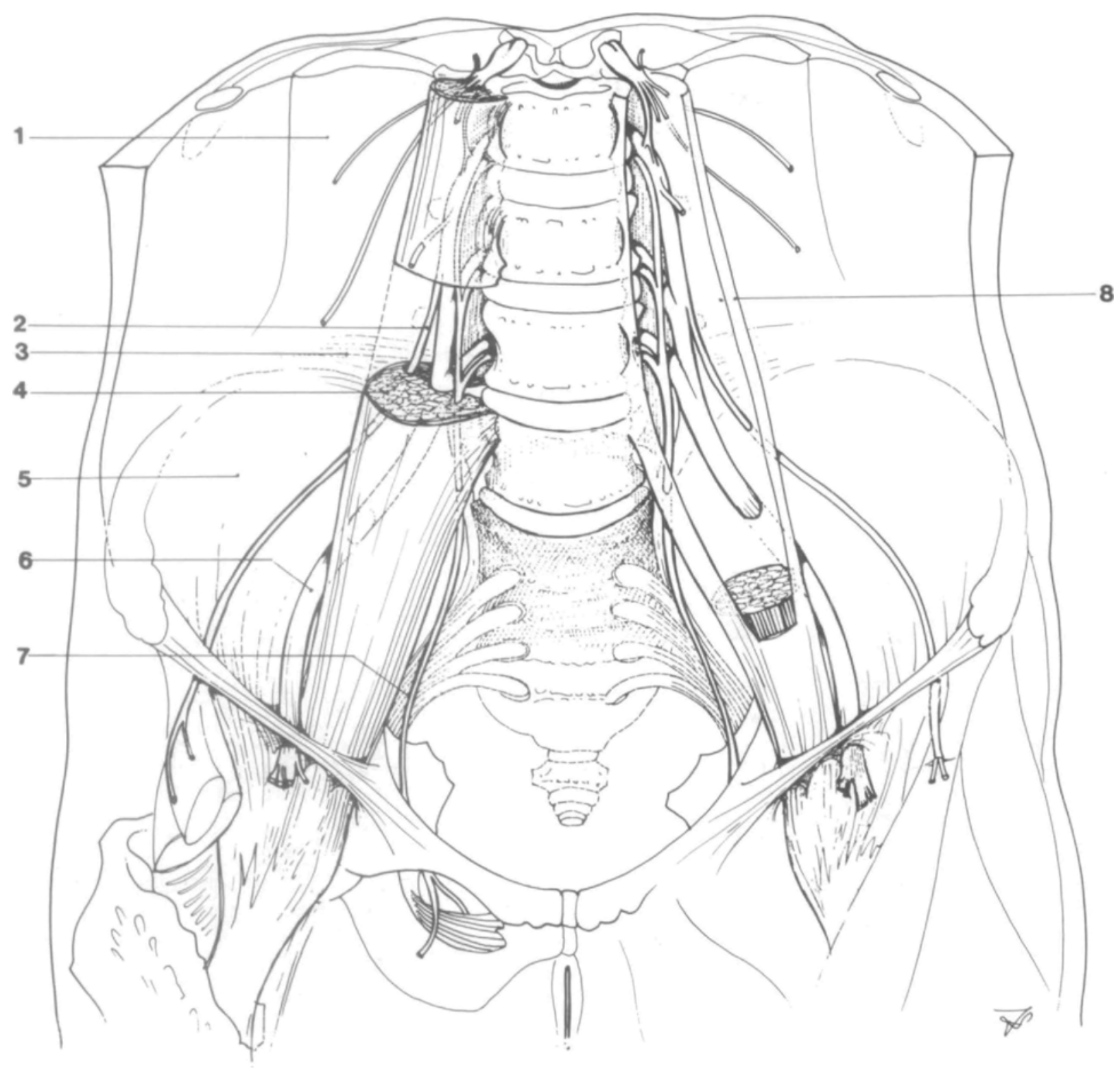

FIGURE 2 Lumbar plexus anatorny. (1) Quadratus lumborum muscle, (2) lateral femoral cutaneous nerve, (3) iliolumbar ligament, (4) psoas major muscle, (5) iliacus muscle, (6) femoral nerve, (7) obturator nerve, (8) psoas major fascia.

inferior margin of the fourth lumbar vertebra. The large posterior divisions of the ventral rami of $\mathrm{L}_{2-3-4}$ united to form the femoral nerve. It was median to the lateral femoral cutaneous nerve. The femoral nerve left the psoas major from its postero-lateral border at the junction of the muscle's upper two thirds and lower third. It then traveled in the gutter between the psoas major and iliacus muscles. The anterior divisions of $\mathrm{L}_{2-3-4}$ were smaller and gave rise to the obturator nerve. The obturator nerve, being median to the femoral nerve, was the innermost nerve of the lumbar plexus. The obturator nerve left the psoas muscle on its internal and posterior side between $L_{5}$ and $S_{1}$. All measurements made following the transverse section can be found in Table I.

During dissection, variations were found concerning the position of the three main nerves of the lumbar plexus within the psoas major (Figure 3). The lateral femoral cutaneous nerve and the femoral nerve were within the psoas major substance at the junction of the posterior third and anterior two-third and in a constant relationship with one another. The obturator nerve localization was more variable. In two of the four cadavers it was in the 
TABLE I Dissection data

\begin{tabular}{lllllllll}
\hline & \multicolumn{8}{c}{ Numbers corresponding to Figure I } \\
\cline { 5 - 10 } Sex & Age & Weight & 1 & 2 & 3 & 4 & 5 & 6 \\
\hline Female & 16 & 50 & 5 & 6 & 3.5 & 5.5 & 3 & 3 \\
Female & 32 & 70 & 6 & 7 & 4 & 5 & 3 & 3 \\
Male & 69 & 62 & 6 & 7 & 4.5 & 6 & 3 & 3.5 \\
Male & 25 & 80 & 7.5 & 9 & 5.2 & 7 & 3.5 & 3.8 \\
\hline
\end{tabular}

All values (except age $=$ years and weight $=\mathrm{kg}$ ) are in $\mathrm{cm}$.

Numbers (corresponding to Figure 1): (1) distance skin/transwerse process $L_{5}$, (2) distance skin/lumbar plexus nervous trunks, (3) distance nervous trunks/median sagital plane, (4) distance psoas major lateral bonder/median sagital plane, (5) psoas major frontal diameter, (6) psoas major median sagital diameter.
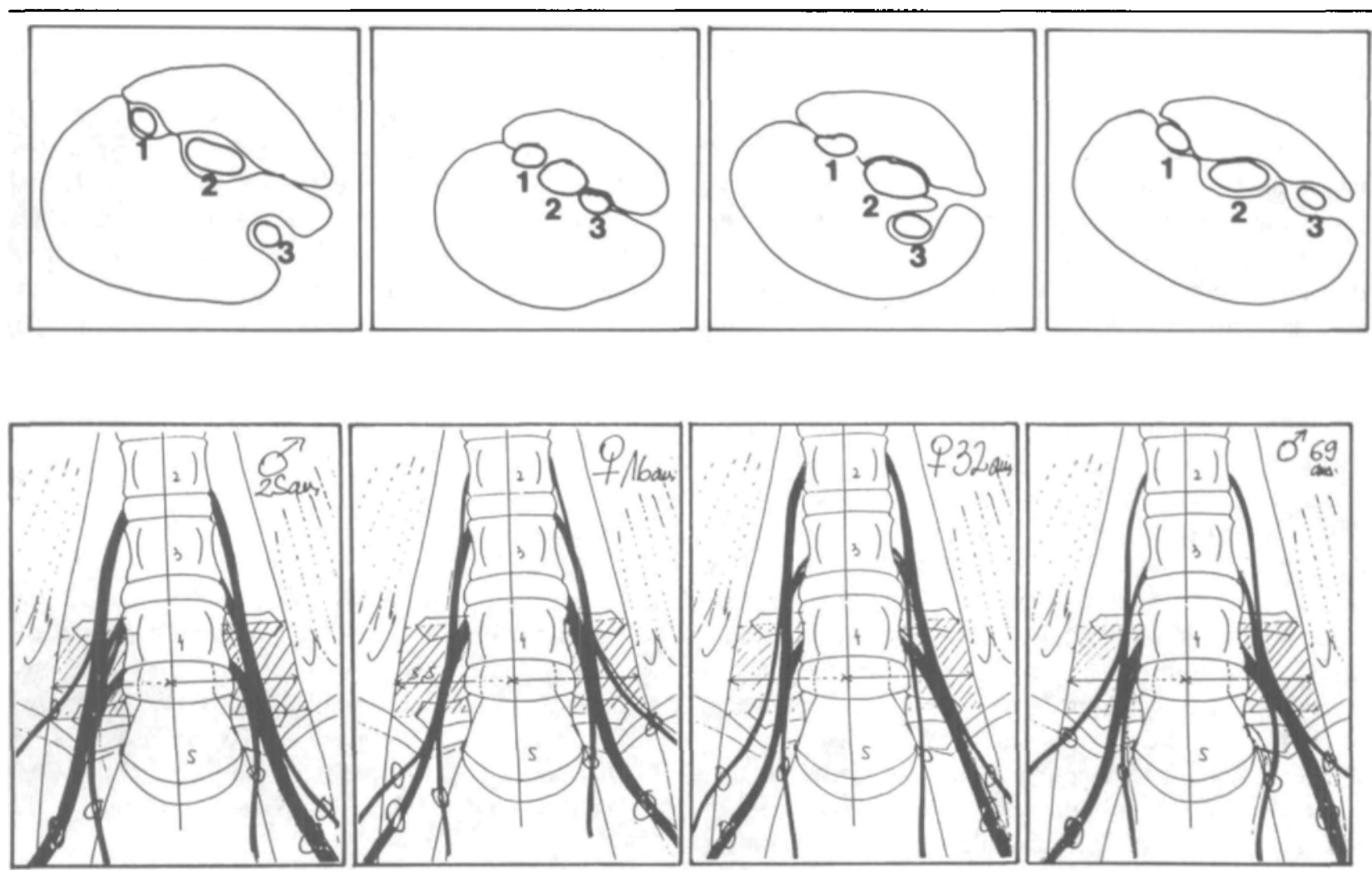

FIGURE 3 Variations in the position of the lumbar plexus main nerves within the psoas major muscle at the $\mathbf{L}_{4-5}$ intervertebral disc level.

same fascial plane as the two other nerves, while in the other two dissections it was in a fold of the psoas major different from the one enclosing the two other nerves.

\section{Computed tomography}

Measurements made from computed tomography show no significant difference between the two sexes (Table II). The mean ( $\pm S D$ ) results are: subcutaneous fat thickness $(2.65 \pm 1.67 \mathrm{~cm})$, intervertebral disc diameter $(5.29$ $\pm 1.21 \mathrm{~cm})$, distance skin to transverse process $(7.02 \pm$
$2.12 \mathrm{~cm}$ ), distance medial aspect psoas to median sagital plane $(2.73 \pm 0.64 \mathrm{~cm})$, distance lateral aspect psoas to median sagital plane $(6.41 \pm 1.61 \mathrm{~cm})$, distance posterior aspect psoas to skin $(7.79 \pm 2.17 \mathrm{~cm})$, and distance anterior aspect psoas to $\operatorname{skin}(11.58 \pm 3.03 \mathrm{~cm})$. The computed tomography reconds did not allow us to identify the femoral nerves within the muscle's substance. However, if the junction of the posterior third and anterior two-third of the psoas major is used as the plane of the femoral nerve, the distance of the femoral nerve to the skin is $9.01 \pm 2.43 \mathrm{~cm}$. 
TABLE II Patient age and computer tomographic data

\begin{tabular}{lcc}
\hline & Female $(n=13)$ & Male $(n=9)$ \\
\hline Age (Yr) & $53.9 \pm 14.9$ & $54.8 \pm 11.4$ \\
Subcutaneous fat thickness & $2.8 \pm 1.6$ & $3.0 \pm 1.9$ \\
Intervertebral disc diameter & $5.3 \pm 0.5$ & $5.7 \pm 0.5$ \\
Distance skin/transverse process & $7.0 \pm 1.3$ & $7.5 \pm 2.2$ \\
Distance medial aspect psoas/median sagital plane & $2.8 \pm 0.3$ & $2.9 \pm 0.2$ \\
Distance lateral aspect psoas/median sagital plane & $6.0 \pm 0.8$ & $7.27 \pm 1.0$ \\
Distance posterior aspect psoas/skin & $7.8 \pm 1.4$ & $8.4 \pm 2.0$ \\
Distance anterior aspect psoas/skin & $11.4 \pm 1.6$ & $12.9 \pm 2.4$ \\
Estimated* distance femoral n./skin & $9.0 \pm 1.4$ & $9.9 \pm 2.1$ \\
\hline
\end{tabular}

All values (except age) are in $\mathrm{cm} \pm$ standard deviation.

*See text: estimated to be at the junction of the posterior third and the anterior two-thirds of the psoas major.

\section{Discussion}

The dissections demonstrate that at the level of $\mathrm{L}_{4-5}$, a site frequently chosen for needle insertion during lumbar plexus block, ${ }^{1,9}$ two of the three main nerves of the lumbar plexus, the lateral femoral cutaneous and the femoral, are within the psoas major muscle. They are inside a thin fascia at the level of the junction of the posterior third and the anterior two-thirds of this muscle. In two of the four dissections the obturator nerve was within its own muscular fold in a plane close to the two other nerves. The clinical consequences of this variable localization on the success rate for the obturator nerve have not been studied. However, it could help to explain the different success rate of obturator nerve block between the 3-in-1 and posterior approach. ${ }^{10}$ The nerves display a fanned-out distribution, the lateral femoral cutaneous being outermost, the obturator nerve innermost and the femoral nerve in-between. It is noteworthy that, despite individual variations (Figure 3 ), the three main nerves were within the psoas major substance in all our dissections.

The computed tomography study was added to the dissection to understand the relationship between the superficial and deep anatomical landmarks used to perform the posterior approach to the lumbar plexus block. A first important measurement is the distance of the femoral nerve from the skin $(9.01 \pm 2.43 \mathrm{~cm})$. Also, because of the distance between the internal border of the psoas muscle and the median sagital plane $(2.73 \pm 0.64 \mathrm{~cm})$, any attempt to reach the plexus by a more medial approach or by a medially oriented needle ${ }^{7}$ can be the cause of a bilateral anaesthesia, be it epidural, spinal or by another mechanism. This does occur; the frequency depending on the technique used. Dalens, ${ }^{9}$ when using Chayen ${ }^{7}$ technique, reported an $88 \%$ incidence of bilateral block, while no bilateral blocks were encountered when using Winnie's' technique. Also, a needle inserted laterally at more than $6.41 \pm 1.61 \mathrm{~cm}$ from the spinal processes may completely miss the psoas major muscle. Insertion of a needle to a depth of more than $11.6 \pm 3.0 \mathrm{~cm}$ can lead to a retroperitoneal injection. There may be a discrepancy between the computed tomography measurements and the distances encountered in clinical practice due to a number of reasons: difference between the orientation of the computed tomography and of needle insertion, patient positioning, individual variations, etc. However, we believe that these data hold an interesting frame of reference to understand the relationships of the structures through which the needle passes to reach the nerves we wish to block.

\section{Conclusion}

Fresh cadaver dissections demonstrated the position of the lumbar plexus within the psoas major muscle substance. The obturator nerve can be separated from the femoral and lateral femoral cutaneous nerves by a muscular fold. Coupled with the computed tomography data, these observations define precisely the relationship of the lumbar plexus with superficial and deep landmarks. Clinical confirmation of these data appears warranted.

\section{Acknowledgments}

The authors wish to thank Jean-Pierre Jean, Department of Radiology, and Dr. Christian Lamoureux, Department of Pathology, for their collaboration in the radiology and dissection parts of our work and Dr. Marcel Jacob, Department of Anaesthesia for reviewing the manuscript.

\section{References}

1 Winnie AP, Ramamurthy $S$, Durrani $Z$, Radonjic $R$. Plexus blocks for lower extremity surgery. Anesthesiology Review 1974; 11-6.

2 Wedel DJ, Brown DL. Nerve blocks. In: Miller RD (Ed.) Anesthesia, 3rd ed., New York: Churchill Livingstone, 1990: 1407-37. 
3 Hovelacque A. Anatomie des Nerfs Crâniens et Rachidiens et du Système Grand Sympathique chez l'Homme. Paris: Doin, 1927.

4 Rouvière H. Anatomie Humaine. 10th ed. Paris: Masson et $\mathrm{Cie}, 1967$.

5 Koritké JG, Sick H. Atlas de Coupes Sériées du Corps Humain. Munich: Urban \& Schwarzenberg, 1982.

6 Davies $D V(E d$.$) . Gray's Anatomy. 34th ed. London:$ Longmans, 1967.

7 Chayen D, Nathan H, Chayen $M$. The psoas compartment block. Anesthesiology 1976; 45: 95-9.

8 Dietemann $J L$, Sick $H$, Wolfram-Gabel $R$, Cruz da Silva $R$, Koritké JG, Wakenheim A. Anatomy and computed tomography of the normal lumbosacral plexus. Neuroradiology 1987; 29: 58-68.

9 Dalens B, Tanguy A, Vanneuville $G$. Lumbar plexus block in children: a comparison of two procedures in 50 patients. Anesth Analg 1988; 67: 750-8.

10 Parkinson SK, Mueller JB, Little WL, Bailey SL. Extent of blockade with various approaches to the lumbar plexus. Anesth Analg 1989; 68: 243-8. 